\title{
Clinical characteristics and phenotype distribution in 10 Chinese patients with $\mathrm{X}$-linked adrenoleukodystrophy
}

\author{
MING-RUI JIA ${ }^{1 *}$, WEN-ZHEN WU ${ }^{2 *}$, CHUAN-MING LI ${ }^{3 *}$, XIAO-HUI CAI ${ }^{4}$, \\ LIN ZHANG ${ }^{5}$, FANG YAN ${ }^{6}$, CHAN ZHU $^{3}$ and MING-HONG GU ${ }^{3}$
}

\begin{abstract}
${ }^{1}$ Department of Pain Management, The Second Hospital of Shandong University, Jinan, Shandong 250033; Departments of ${ }^{2}$ Emergency Internal Medicine and ${ }^{3}$ Pain Management; ${ }^{4}$ Injury Department of Orthopedics, Shanghai Jiading Hospital of Traditional Chinese Medicine, Shanghai 201800; Departments of ${ }^{5}$ Intensive Care Unit and ${ }^{6}$ Pain Management, Shandong Provincial Hospital Affiliated to Shandong University, Jinan, Shandong 250021, P.R. China
\end{abstract}

Received August 26, 2018; Accepted April 5, 2019

DOI: $10.3892 /$ etm.2019.7804

\begin{abstract}
X-linked adrenoleukodystrophy (X-ALD) is the most frequent type of inherited demyelinating peroxisomal disease caused by mutations in the ATP binding cassette subfamily D member 1 (ABCD1) gene. The rate of early recognition and genetic diagnosis of X-ALD remains low due to its variable clinical manifestations. The present study summarized the clinical features Chinese X-ALD patients and performed a follow-up study to further precisely characterize this disease. A total of 10 patients diagnosed with X-ALD between 1994 and 2016 at Shandong Provincial Hospital Affiliated to Shandong University (Jinan, China) were included in the present study. Through reviewing their medical records and performing telephone follow-ups, the clinical features, biochemical laboratory data, brain images, treatments and long-term outcomes were retrospectively summarized. Mutation analysis of the ABCD1 gene was performed in certain patients. Most of the patients $(8 / 10)$ had the childhood cerebral form of X-ALD. One patient presented with the olivo-ponto-cerebellar form, the rarest form of X-ALD. In all patients, brain magnetic resonance images revealed abnormalities with typical T2-weighted hyperintensity. Analysis of very long chain fatty acid revealed high plasma levels of hexacosanoic acid in all patients. Increased adrenocorticotropic hormone, decreased cortisol and neurophysiological manifestations were also observed. Three different mutations of the ABCD1 gene were
\end{abstract}

Correspondence to: Professor Ming-Hong $\mathrm{Gu}$ or Professor Chan Zhu, Department of Pain Management, Shanghai Jiading Hospital of Traditional Chinese Medicine, 222 Bole Road, Shanghai 201800, P.R. China

E-mail: 273050718@qq.com

E-mail: zhuchan2009@163.com

*Contributed equally

Key words: ABCD1 gene, leukodystrophy, very long chain fatty acids, X-linked adrenoleukodystrophy identified in the 3 patients subjected to genotyping. During the follow-ups, most patients took neurotrophic drugs and received hydrocortisone replacement when required. One patient received a hematopoietic stem cell transplantation, but died 1 year following the transplantation. Chronic myelopathy and peripheral neuropathy progressed with time, gradually leading to a vegetative state or paralysis within several years of clinical symptom onset. In conclusion, male patients with adrenocortical insufficiency should be further investigated for $\mathrm{X}$-ALD. Early detection is critical to prevent the progression of X-ALD with mutation analysis of ABCD1 the most accurate method to confirm diagnosis.

\section{Introduction}

X-linked adrenoleukodystrophy (X-ALD; Online Mendelian Inheritance in Man no. 300100) is the most common type of inherited peroxisomal disorder and monogenic leukodystrophy that occurs in $\sim 1$ in 17,000 live male births with no apparent difference in the prevalence among different ethnicities (1). The clinical manifestation is highly variable, including progressive demyelination of the central and peripheral nervous systems, adrenal insufficiency, and accumulation of very long chain fatty acids (VLCFAs) in body fluid and tissues (2). Adrenomyeloneuropathy (AMN) and the cerebral form of X-ALD (CALD) are the two most common phenotypes of X-ALD. CALD includes the childhood (CCALD), adolescent (AdolCALD) and adult forms with onset ages of $3-10,11-20$ and $>21$ years, respectively $(3,4)$. CCALD, the most fatal phenotype, is associated with rapidly progressive inflammatory brain demyelination and frequently leads to total disability within 3 years.

$\mathrm{X}$-ALD is caused by mutations in the ATP-binding cassette protein subfamily D1 (ABCD1; GenBank accession no., NM_000033) gene, which is located on the $\mathrm{X}$ chromosome at Xq28 (5). The gene contains 10 exons and encodes the ALD protein (ALDP), which is located in the peroxisome membrane and has an important role in transporting VLCFAs from the cytosol into the peroxisome for degradation (6). Functional defects of ALDP block this transport, resulting in impaired degradation of VLCFAs and subsequent 


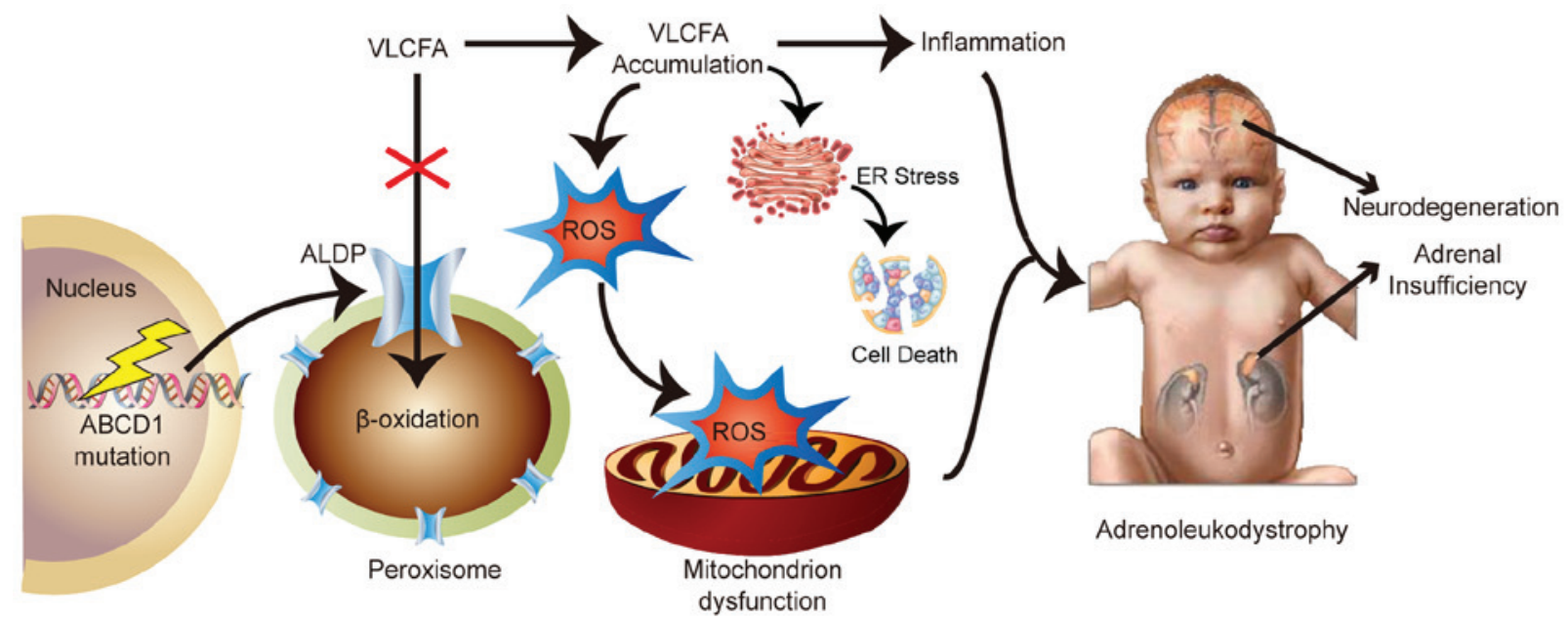

Figure 1. Schematic illustrating the molecular mechanisms associated with the pathogenesis of X-ALD. The mutated ABCD1 gene encodes a defective ALDP that blocks VLCFA transport to the peroxisome. This defective transport leads to the impaired degradation of VLCFAs and subsequent accumulation of VLCFA in mainly the white matter and axons of the central nervous system and the adrenal system. Subsequently, mitochondrial dysfunction evoked by oxidative stress, ER stress and lipid-induced cell death are caused by the accumulation of VLCFA. Finally, the nervous system and the adrenal cortex are primarily affected, leading to neurodegeneration and adrenocortical insufficiency. ABCD1, ATP binding cassette subfamily D member 1; ER, endoplasmic reticulum; ROS, reactive oxygen species; ALDP, adrenoleukodystrophy protein; VLCFA, very long chain fatty acid.

accumulation of VLCFAs in cells, tissues and organs, mainly in the white matter and axons of the central nervous system and the adrenal glands (7). The accumulation of VLCFAs has a toxic effect by evoking mitochondrial dysfunction and by causing the breakdown of cell membranes as a result of oxidative stress. The molecular mechanisms associated with the pathogenesis of X-ALD are summarized in Fig. 1. To date, 2,692 mutations in the ABCD1 gene have been reported and recorded in the ALD database (https://adrenoleukodystrophy.info/). Mutation analysis of the ABCD1 gene is of great value to make a definite diagnosis and provide genetic counseling to families $(8,9)$.

Various Chinese studies have reported on X-ALD (10-13); however, the rate of early identification and genetic diagnosis remain low. Identification of more novel mutations in the ABCD1 gene and investigation of clinical characteristics and phenotype distribution in the Chinese population may expand the current knowledge on X-ALD and ideally provide improved references for genomics-based treatments and prevention strategies. The present study performed a retrospective analysis of 10 Chinese patients with X-ALD diagnosed during infancy or adulthood and evaluated their long-term outcomes.

\section{Materials and methods}

Patients. All patients were diagnosed with X-ALD at the Shandong Provincial Hospital Affiliated to Shandong University (Jinan, China) between July 1994 and June 2016. The diagnosis was based on neurological symptoms, including pyramidal symptoms, impaired hearing and language skills, behavioral changes, epilepsy, cognition impairment, inflammatory brain demyelination on brain magnetic resonance imaging (MRI), adrenal dysfunction, increased concentration of plasma VLCFA and gene mutation analysis (14).

Clinical data analysis. Patient records in electronic format and on paper were reviewed for clinical features, biochemical parameters, diagnostic images, molecular genetic tests, treatments and long-term outcomes. The disease phenotype, including symptoms and laboratory results, were evaluated at the first presentation. Telephone consultation was also performed when required. The clinical characteristics of one of these patients have been previously reported (15). To facilitate the diagnosis of the patients, the plasma levels of adrenocorticotropic hormone (ACTH) and cortisol were determined by electrochemiluminescence immunoassay (Elecsys ACTH and Cortisol II, Roche Diagnostics). Plasma VLCFA levels were determined by gas chromatography/mass spectrometry (QP5050A; Shimadzu). Electromyography (EMG) and nerve conduction velocities (NVC) were measured using Keypoint Amplifier (cat. no. 9031C0762; Dantec Ltd.). Lumbar puncture was conducted by an experienced neurological physician and cerebrospinal fluid (CSF) analyses were performed routinely.

Mutation analysis of the ABCDI gene. Genomic DNA was prepared from peripheral blood leukocytes sampled from the patients using a genomic DNA extraction kit (QIAamp Blood DNA Mini kit; Qiagen). Previously published primer sequences and amplifying conditions were used to amplify the whole ABCD1 gene (6). The complete coding sequence and all of the exon-intron boundaries of the ABCD1 gene were subjected to automated sequencing on an ABI 3500 DNA sequencer (Thermo Fisher Scientific, Inc.). The analyzed sequences were compared to the corresponding wild-type sequences of ABCD1 in GenBank (NM_000033). Direct sequencing of the ABCD1 gene from 100 control samples (age, 38.7 \pm 9.3 years; male/female ratio, 1.2:1.0) was also performed to rule out any polymorphisms. All of the controls were recruited from the examination center of Shandong Provincial Hospital (Jinan, China) from April 2015 to September 2015. All subjects provided written informed consent for the collection and use of their blood samples for the purposes of research and for the publication of their results.

Follow-up examinations. Follow-up examinations were approved by the ethics committee. All patients were reviewed 
Table I. Demographic data and clinical characteristics of 10 patients with X-ALD.

\begin{tabular}{|c|c|c|c|c|c|c|}
\hline $\begin{array}{l}\text { Case } \\
\text { no. }\end{array}$ & $\begin{array}{c}\text { Age at } \\
\text { onset } \\
\text { (years) }\end{array}$ & $\begin{array}{c}\text { Age at } \\
\text { diagnosis } \\
\text { (years) }\end{array}$ & Clinical features & $\begin{array}{l}\text { Family } \\
\text { history }\end{array}$ & $\begin{array}{c}\text { Adrenal } \\
\text { dysfunction }\end{array}$ & Phenotype \\
\hline 1 & 3 & 3 & $\begin{array}{l}\text { Vomiting, skin pigmentation, pyramidal symptoms, } \\
\text { adrenal crisis }\end{array}$ & - & + & CCALD \\
\hline 2 & 4 & 6 & $\begin{array}{l}\text { Hearing impairment, walking instability after a head } \\
\text { blow, skin pigmentation, pyramidal symptoms }\end{array}$ & - & + & CCALD \\
\hline 3 & 4 & 5 & $\begin{array}{l}\text { Visual impairment, hearing impairment, hallucination } \\
\text { of soliloquy, pyramidal symptoms }\end{array}$ & + & - & CCALD \\
\hline 4 & 7 & 7 & $\begin{array}{l}\text { Visual impairment, unsteady gait, memory decline, } \\
\text { skin pigmentation, pyramidal symptoms, adrenal crisis }\end{array}$ & - & + & CCALD \\
\hline 5 & 3 & 3 & Convulsion, unsteady gait, aphasia, pyramidal symptoms & - & - & CCALD \\
\hline 6 & 6 & 7 & Visual impairment, easy to tumble down, skin pigmentation & - & + & CCALD \\
\hline 7 & 6 & 6 & Convulsion after a head blow, skin pigmentation & + & + & CCALD \\
\hline 8 & 8 & 8 & $\begin{array}{l}\text { Visual impairment, convulsion, memory decline, skin } \\
\text { pigmentation, pyramidal symptoms }\end{array}$ & - & + & CCALD \\
\hline 9 & 12 & 12 & $\begin{array}{l}\text { Unsteady gait, impaired language skills, pyramidal } \\
\text { symptoms }\end{array}$ & - & + & AdolCALD \\
\hline 10 & 29 & 30 & $\begin{array}{l}\text { Ataxia, impaired language skills, difficult swallowing, } \\
\text { skin pigmentation, pyramidal symptoms }\end{array}$ & - & + & $\begin{array}{l}\text { Olivo-ponto- } \\
\text { cerebellar ALD }\end{array}$ \\
\hline
\end{tabular}

by telephone every six months following the first diagnosis. Patients were seen at our hospital for examination and treatment once every year or if there were any special circumstances, such as 2 patients who had communication problems. The symptoms, treatments, occurrence of potential complications and the evolution of the disease were closely monitored.

Statistical analysis. Data were analyzed using SPSS v.17.0 (SPSS, Inc., Chicago, IL, USA). Values are expressed as the median (range) or the mean \pm standard deviation. Differences between means were compared using one-way analysis of variance for multiple comparisons and Dunnett's test was performed as a post-hoc test. All of the calculated P-values were two-sided and $\mathrm{P}<0.05$ was considered to indicate a statistically significant difference.

\section{Results}

Clinical characteristics and phenotypes. A total of 10 patients (all males) were included in the present retrospective study. They were all offspring of non-consanguineous marriages and were born after an uneventful full-term pregnancy. The median age at onset of disease was 8.2 years old (range, 3-29 years). According to the clinical symptoms of different phenotypes described previously $(2,3)$, most of the patients had CCALD at an age of 3-10 years $(n=8)$ and 1 patient had AdolCALD (age, 12 years old). Of note, one 29-year old patient had adult-onset olivo-ponto-cerebellar X-ALD, a rare form of
X-ALD (prevalence, 1-2\% of X-ALD) with clinical features of cerebellar and brainstem involvement in adolescence or adulthood (4). The mean duration of follow-up was 9.05 \pm 5.20 years The phenotypes, clinical features, family history and adrenal dysfunction of the patients are summarized in Table I. Paraclinical and laboratory studies were performed and the results are summarized in Table II.

Two of the patients have a family history of X-ALD or Addison's disease (20\%). Most of the family members refused any clinical genetic investigations thus far; however, a certain number of carriers in the respective families would be expected. All patients were symptomatic at the time of the diagnosis, and none of them was diagnosed in the asymptomatic stage based on a positive family history of X-ALD. Most patients $(8 / 10)$ had the manifestation of adrenal dysfunction characterized as hyperpigmentation in lips, gums and even the whole body owing to high levels of ACTH. Neurodevelopmental regression and behavioral problems were common. The clinical manifestations included visual impairment $(n=4)$, unsteady gait $(n=3)$, memory decline $(n=2)$, impaired language skills $(n=2)$ and cognitive decline $(n=3)$.

Brain images. The brain images of all patients exhibited abnormalities. T2-weighted hyperintensity (caused by neurocyte demyelination) on MRI was observed in the cerebrum of 5 patients, and 6 patients had lesions in the brainstem, internal capsule and/or cerebellum. The initial abnormalities usually start from the splenium of the corpus callosum and then spread 


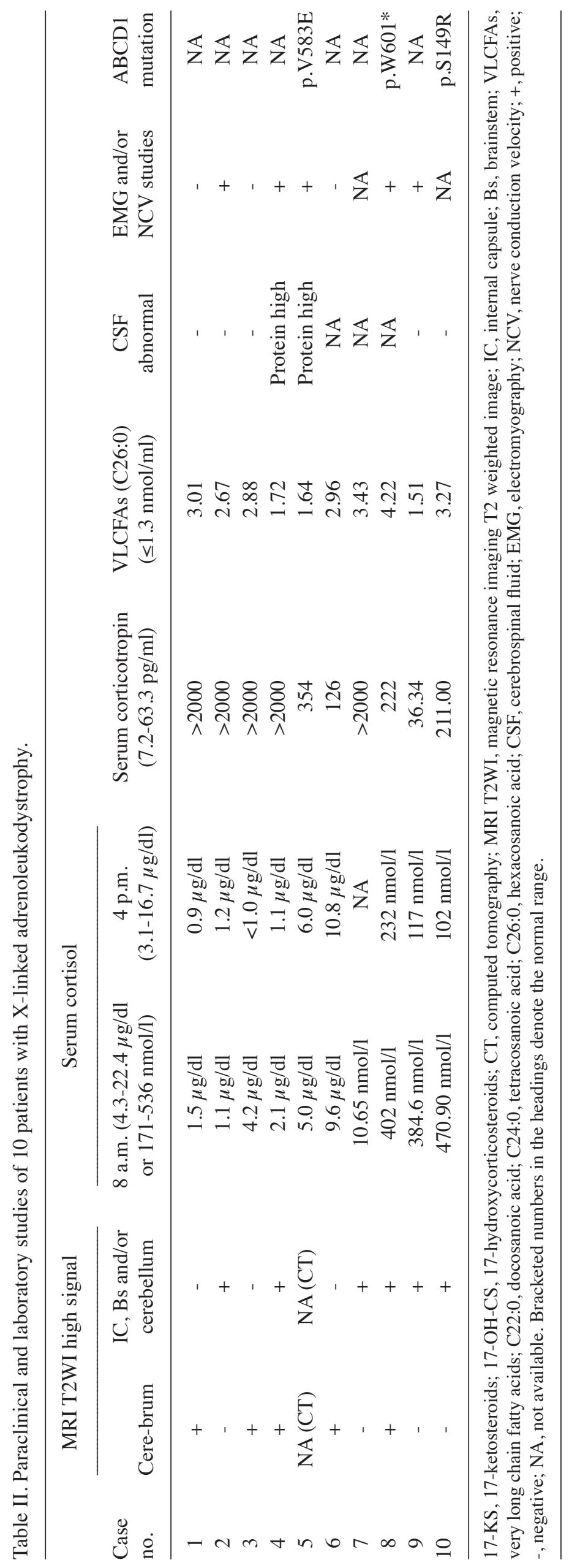



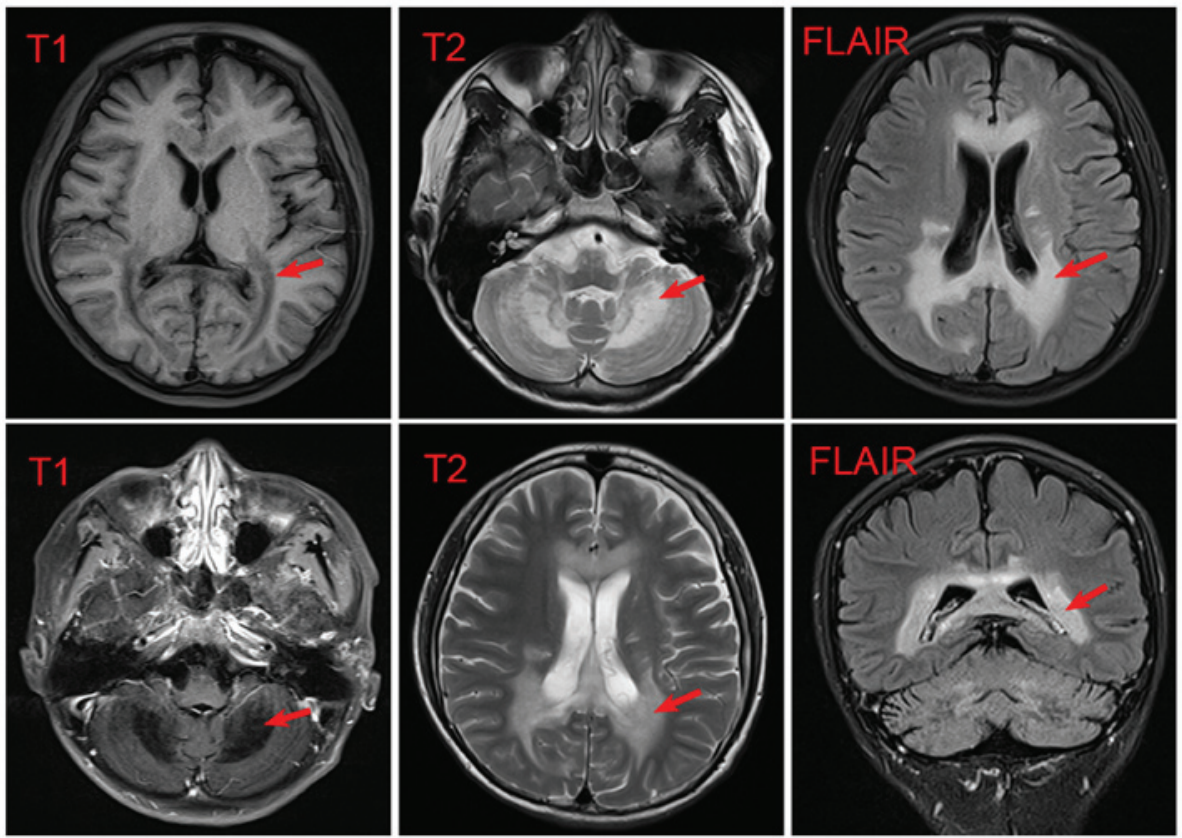

Figure 2. Magnetic resonance images of the brain of Case no. 10 exhibiting characteristic extensive white matter changes. T1-weighted low-signal-intensity, T2-weighted high-signal-intensity and T2 FLAIR high-signal-intensity lesions (highlighted with red arrow) were present symmetrically in the bilateral white matter around the lateral ventricle, genu and splenium of the corpus callosum as well as in the cerebellum. The demyelinating lesions are extensive and correspond to an advanced disease stage. FLAIR, fluid-attenuated inversion recovery.

into the adjacent white matter of the parieto-occipital lobes (13). The MRI of 1 patient was not available; however, his cerebral CT exhibited diffuse atrophy and diffuse hypodensities in the white matter and posterior horn of the lateral ventricles and centrum semiovale. All of the cerebral demyelinating lesions were symmetric. The most severe demyelination (case no. 10) is presented in Fig. 2. These images showcase the characteristics on MRI for the diagnosis of the disorder. Of note, MRI is currently used in China to monitor young males who have a high risk of developing cerebral X-ALD (16).

Biochemical analysis. Plasma hexacosanoic acid (C26:0) levels were determined at the time of diagnosis. VLCFA analysis revealed high plasma levels of C26:0 in all patients. The median (range) levels of C26:0 were 2.92 (1.51-4.22) nmol/ml (normal, $\leq 1.3 \mathrm{nmol} / \mathrm{ml}$ ). There was no significant difference in the elevated VLCFA levels among patients with different phenotypes of X-ALD ( $\mathrm{P}>0.05)$.

At the time of the first presentation at our hospital, none of the patients had previously received hydrocortisone therapy. High levels of ACTH and low levels of cortisol (measured at 8 am and at $4 \mathrm{pm}$ ) were present in half of the patients, which were indicative of a state of adrenocortical insufficiency.

Paraclinical and laboratory results. Of the 10 patients, 8 were subjected to neurophysiological examination, of whom 5 patients presented with an aberration on EMG and/or in NVC, which included moderate sensorimotor peripheral neuropathy in the extremities with predominantly axonal changes. There was no association between neurophysiological results and the severity of the symptomatic status. Other electrophysiological examinations, including electrocardiogram, visual evoked potentials, brainstem auditory evoked potentials and somatosensory evoked potentials, were in the normal ranges (data not shown).

CSF analyses were mostly normal; two out of 10 patients presented with slightly elevated CSF protein levels above the normal range.

Mutations in the ABCDl gene. A total of 3 different mutations were identified in 3 patients, including 2 missense mutations (p.V583E, p.S149R) and 1 nonsense mutation (p.W601*). The p.S149R mutation has been identified in the same patient, which has already been reported in a previous study by our group (14), and this mutation was likely to be pathogenic based on the in silico analysis prediction tools and elevated level of VLCFA. The other two mutations were also described previously (17). The remaining 7 patients denied genetic testing.

Therapy and follow-up. Table III summarizes the therapy and follow-up of patients with X-ALD. During hospitalization, most patients received neurotrophic drugs, and if adrenal insufficiency was present, hydrocortisone replacement therapy was initiated. All of the patients were requested to follow a low-VLCFA diet. Occasionally, medical therapies were provided with supportive care by rehabilitation physicians.

During follow-up, X-ALD phenotypes progressed with time. With time, almost all of the patients are at risk of developing neurologic (CALD, AMN) or endocrinologic (Addison's disease) symptoms. In most of these patients (6/10), chronic myelopathy and peripheral neuropathy progressed with time, gradually leading to a vegetative state or paralysis within several years of clinical symptom onset. Case no. 8 received a hematopoietic stem cell transplantation (HSCT) at the advanced stage of the disease. He exhibited neurological improvement for 3 months post-transplantation. However, after another 3 months, 
Table III. Treatment and follow-up of 10 patients with X-linked adrenoleukodystrophy.

\begin{tabular}{|c|c|c|c|}
\hline $\begin{array}{l}\text { Case } \\
\text { no. }\end{array}$ & $\begin{array}{l}\text { Duration of } \\
\text { follow-up (years) }\end{array}$ & Treatment & Outcome \\
\hline 1 & 15.3 & $\begin{array}{l}\text { Anti-convulsants, penicillin, neurotrophic drugs, hormone replacement } \\
\text { therapy, low-VLCFA diet, ATP, CoA }\end{array}$ & Addison only \\
\hline 2 & 5.6 & Penicillin, neurotrophic drugs, hormone replacement therapy, ATP, CoA & Disability \\
\hline 3 & 12.3 & $\begin{array}{l}\text { Anti-convulsants, penicillin, neurotrophic drugs, hormone replacement } \\
\text { therapy, low-VLCFA diet, ATP, CoA }\end{array}$ & Dementia \\
\hline 4 & 12 & Neurotrophic drugs, hormone replacement therapy, ATP, CoA & Dementia, disability \\
\hline 5 & 16.1 & Penicillin, neurotrophic drugs, hormone replacement therapy, ATP, CoA & Addison only \\
\hline 6 & 4.8 & Ceftriaxone, neurotrophic drugs, hormone replacement therapy, ATP & Dementia \\
\hline 7 & 11.5 & $\begin{array}{l}\text { Azithromycin, neurotrophic drugs, hormone replacement therapy, } \\
\text { low-VLCFA diet }\end{array}$ & Addison only \\
\hline 8 & 2.4 & Neurotrophic drugs, hormone replacement therapy, ATP, HSCT & Death \\
\hline 9 & 8.9 & No treatment & Asymptomatic \\
\hline 10 & 1.6 & Neurotrophic drugs, hormone replacement therapy & Exacerbation \\
\hline
\end{tabular}

HSCT, hematopoietic stem cell transplantation; VLCFA, very long chain fatty acid.

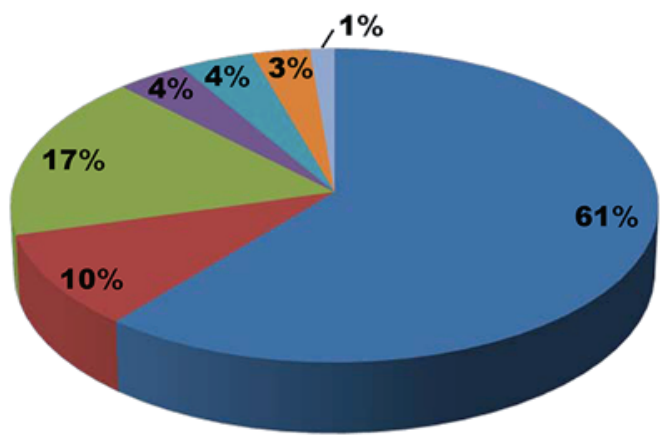

aissense mutations

- Nonsense mutations

Frame shift mutations

- Amino acid insertions/deletions

Splice site mutations

- One or more exons deleted

a Benign variants

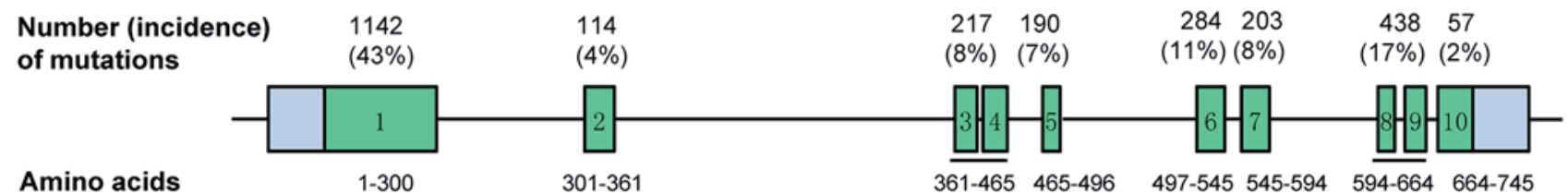

Figure 3. Statistics of ABCD1 mutations (upper panel) and genomic structure of the human ABCD1 gene and incidence of mutations in each genomic amplicon (lower panel). ABCD1, ATP binding cassette subfamily D member 1.

his symptoms gradually deteriorated with progressive mental disorder and he died 1 year after the transplantation.

\section{Discussion}

In the present study, the clinical data of 10 Chinese patients with $\mathrm{X}$-ALD were retrospectively reviewed and a follow-up study was performed. Most of the patients (8/10) had CCALD. One patient presented with the olivo-ponto-cerebellar form, the rarest form of ALD. The brain MRIs of all patients exhibited abnormalities with typical T2-weighed hyperintensity. VLCFA analysis revealed high plasma levels of C26:0 in all patients. Increased ACTH, decreased cortisol and neurophysiological manifestations were also noted. In 3 of the patients, 3 different mutations of the ABCD1 gene were identified. During follow-up, most patients took neurotrophic drugs and received hydrocortisone replacement when required. Furthermore, one patient received HSCT. The present results may enhance the current understanding of this disorder and provide valuable information for the future prevention and therapies for this disorder.

Of note, one of the patients of the present study had the adult-onset olivo-ponto-cerebellar form, the rarest form of X-ALD, which is the same patient that has been previously described by our group (14). Approximately $88 \%$ of olivo-ponto-cerebellar X-ALD patients are from Korea and Japan (18). One case was reported in Taiwan (19). To the best of our knowledge, the present case was the first case from the Chinese mainland that was ever reported. Genetic analysis confirmed the diagnosis based on a mutation (c.447T $>$ A; p.S149R) in the ABCD1 gene detected in this patient. A further 
molecular functional study may provide more insight into the underlying mechanisms of this disease.

In agreement with a previous study from China, the majority of the CCALD cases were of CCALD (80\%) (20). Almost all of these patients had adrenocortical insufficiency (Addison's disease), which may be the only clinical manifestation that was apparent prior to the onset of cerebral symptoms $(21,22)$. According to certain studies, adrenocortical insufficiency was the first symptom of X-ALD in $38 \%$ of patients (23), adrenal symptoms appeared prior to neurological symptoms in $92 \%$ of cases of CCALD (24) and the incidence was 100\% in those patients whose adrenal insufficiency became evident prior to the age of 7.5 years (25). These results are clinically significant. In male patients presenting with Addison's disease, including those who only have a glucocorticoid deficiency, X-ALD should be taken into consideration, and plasma VLCFA levels should be determined (14). The requirement to investigate X-ALD and plasma VLCFA levels is particularly important in patients whose adrenal insufficiency manifested prior to the age of 7.5 years.

However, throughout the world, CCALD is not the most common phenotype of ALD (26). The fact that most studies from China report on a higher percentage of CCALD compared to other countries may be due to numerous patients with milder phenotypes remaining undiagnosed, particularly those with AMN, the most common phenotype (4). Therefore, a bias regarding the detection of phenotypes may exist in China.

In addition, X-ALD should be considered in young and adult males presenting with cognitive and neurological symptoms with white matter lesions on brain MRI. For females with X-ALD, mutation analysis of the ABCD1 gene should also be performed, as plasma VLCFA levels were reported to be normal in $15 \%$ of females with X-ALD (3). A more recent study revealed that females with X-ALD with normal plasma VLCFA levels had elevated C26:0-lysophosphatidylcholine (C26:0-lyso-PC) according to dried blood spot analysis (27). Analysis of C26:0-lyso-PC is cheaper than conventional plasma VLCFA analysis and may be performed using dried bloodspots, which are generated on Guthrie paper; they may be stored at room temperature and sent to diagnostic centers. Therefore, C26:0-lyso-PC analysis should be added to the routine biochemical array of diagnostic tests for peroxisomal disorders. Regarding the diagnosis, MRI was observed to be more sensitive than CT scan. MRI exhibited a larger area of abnormality, particularly when there was anincreased signal intensity on T2-weighted and fluid-attenuated inversion recovery sequences in the splenium of the corpus callosum and white matter of the parieto-occipital lobes (28).

Follow-up of patients with X-ALD is important for two reasons: i) Early detection of adrenocortical insufficiency and ii) early detection of CALD for the purpose of intervention by allogeneic HSCT (25). There are no unified guidelines for the management of X-ALD and to date, HSCT is considered to be the only effective approach to prevent the progression of cerebral demyelination in X-ALD (29). It is recommended to perform HSCT an early stage of the disease to accomplish a prolonged beneficial effect. Thus, it is required to diagnose $\mathrm{X}$-ALD as early as possible, since treatment, including allogenic HSCT in the early stage (29) and endocrine replacement therapy for adrenocortical insufficiency, is applicable in certain cases. At present, there is no definitive cure for X-ALD. Pedigree studies, as well as identification of individuals at high risk, are required to establish an early preventive treatment plan involving genetic counseling and prevention of disease development in mutation carriers, to provide suitable professional advice for each individual patient.

$\mathrm{X}$-ALD is an inherited peroxisomal disorder due to mutations in the ABCD1 gene (2). According to the ALD database, 2,692 different mutations in the ABCD1 gene have been detected thus far, of which 806 mutations (30\%) appear to be nonrecurrent. Missense mutations, accounting for $61 \%$, are the most common type among ABCD1 mutations. Other mutations include frameshift mutations (17\%), nonsense mutations (10\%), amino acid insertions or deletions (4\%; Fig. 3). The distribution of mutations is imbalanced. Of the mutations, $50 \%$ occurred in exon 1, which encodes the transmembrane domain of the ABCD1 protein. Another clustering of mutations is in exons 6-9, which encode the ATP binding domain of ALDP (3). However, the spectrum of ABCD1 mutations may exhibit geographical distribution differences in different ethnicities. Genotype-phenotype association analyses have been performed ever since the X-ALD gene was identified in 1993. However, no genotype-phenotype correlation has been detected $(26,30)$.

The major limitation of the present study is the absence of screening for mutations in the remaining patients. Another limitation involved the communication difficulties during telephone interview because some of the patients had problems with their hearing, communication and perception. This may bring some bias on the description of the symptoms.

In conclusion, the present retrospective study of 10 Chinese patients with X-ALD suggests that it is critical to investigate $\mathrm{X}$-ALD in male patients with adrenocortical insufficiency. Furthermore, it is essential to diagnose patients early, since treatment is available in certain cases. In a future study, more X-ALD patients will be recruited to establish a prospective study cohort. By performing follow-ups, the clinical characteristics, development and prognosis of the disease will be explored.

\section{Acknowledgements}

Not applicable.

\section{Funding}

The present study was supported by grants from the National Natural Science Foundation (grant nos. 81500608 and 81500607).

\section{Availability of data and materials}

The datasets generated and/or analyzed during the current study are available from the corresponding author on reasonable request.

\section{Authors' contributions}

The present study was carried out in collaboration between all authors. CZ and MG conceived and supervised the project, and also revised the manuscript content. MJ and WW analyzed the data, interpreted the results and wrote the manuscript. CL designed methods, collected data and contributed equally to this 
study. XC performed the statistical analysis. LZ and FY participated in the design of the study. All authors read and approved the final manuscript.

\section{Ethical approval and consent to participate}

The institutional review board of Shandong Provincial Hospital Affiliated to Shandong University (Jinan, China) approved the study protocol, and written informed consent was obtained from the patients or their guardians. The ethics committee of Shandong Provincial Hospital affiliated to Shandong University (Jinan, China) approved the retrospective review of the patients' medical records and licensed the records for research purposes only. All methods were performed in accordance with the relevant guidelines and regulations.

\section{Patient consent for publication}

Not applicable.

\section{Competing interests}

The authors declare that they have no competing interests.

\section{References}

1. Bezman L, Moser AB, Raymond GV, Rinaldo P, Watkins PA, Smith KD, Kass NE and Moser HW: Adrenoleukodystrophy: Incidence, new mutation rate, and results of extended family screening. Ann Neurol 49: 512-517, 2001.

2. Kemp S, Berger J and Aubourg P: X-linked adrenoleukodystrophy: Clinical, metabolic, genetic and pathophysiological aspects. Biochim Biophys Acta 1822: 1465-1474, 2012.

3. Moser HW, Mahmood A and Raymond GV: X-linked adrenoleukodystrophy. Nat Clin Pract Neurol 3: 140-151, 2007.

4. Kemp S, Pujol A, Waterham HR, van Geel BM, Boehm CD, Raymond GV, Cutting GR, Wanders RJ and Moser HW: ABCD1 mutations and the X-linked adrenoleukodystrophy mutation database: Role in diagnosis and clinical correlations. Hum Mutat 18 499-515, 2001.

5. Mosser J, Douar AM, Sarde CO, Kioschis P, Feil R, Moser H, Poustka AM, Mandel JL and Aubourg P: Putative X-linked adrenoleukodystrophy gene shares unexpected homology with $\mathrm{ABC}$ transporters. Nature 361: 726-730, 1993.

6. Boehm CD, Cutting GR, Lachtermacher MB, Moser HW and Chong SS: Accurate DNA-based diagnostic and carrier testing for X-linked adrenoleukodystrophy. Mol Genet Metab 66: 128-136, 1999

7. Singh I, Moser HW, Moser AB and Kishimoto $Y$ Adrenoleukodystrophy: Impaired oxidation of long chain fatty acids in cultured skin fibroblasts an adrenal cortex. Biochem Biophys Res Commun 102: 1223-1229, 1981.

8. Pereira Fdos S, Matte U, Habekost CT, de Castilhos RM, El Husny AS, Lourenco CM, Vianna-Morgante AM, Giuliani L, Galera MF, Honjo R, et al: Mutations, clinical findings and survival estimates in South American patients with X-linked adrenoleukodystrophy. PLoS One 7: e34195, 2012.

9. Dvoráková L, Storkánová G, Unterrainer G, Hujová J, Kmoch S, Zeman J, Hrebicek M and Berger J: Eight novel ABCD1 gene mutations and three polymorphisms in patients with X-linked adrenoleukodystrophy: The first polymorphism causing an amino acid exchange. Hum Mutat 18: 52-60, 2001.

10. Wang Z, Yan A, Lin Y, Xie H, Zhou C and Lan F: Familial skewed $\mathrm{x}$ chromosome inactivation in adrenoleukodystrophy manifesting heterozygotes from a Chinese pedigree. PLoS One 8: e57977, 2013.

11. Ping LL, Bao XH, Wang AH, Pan H, Wu Y, Xiong H, Jiang YW, Qin J and Wu XR: Clinical features and genotype-phenotype studies of 89 Chinese patients with X-linked adrenoleukodystrophy. Zhonghua Er Ke Za Zhi 45: 203-207, 2007 (In Chinese).

12. Ping LL, Bao XH, Wang AH, Pan H, Wu Y, Xiong H, Zhang YH, Jiang YW, Qin J and Wu XR: The genotype and phenotype studies of 40 Chinese patients with X-linked adrenoleukodystrophy (X-ALD). Beijing Da Xue Xue Bao Yi Xue Ban 38: 66-70, 2006.
13. Chu SS, Ye J, Zhang HW, Han LS, Qiu WJ, Gao XL and Gu XF: Eight novel mutations in the ABCD1 gene and clinical characteristics of 25 Chinese patients with X-linked adrenoleukodystrophy. World J Pediatr 11: 366-373, 2015

14. Engelen M, Kemp S, de Visser M, van Geel BM, Wanders RJ, Aubourg P and Poll-The BT: X-linked adrenoleukodystrophy (X-ALD): Clinical presentation and guidelines for diagnosis, follow-up and management. Orphanet J Rare Dis 7: 51, 2012.

15. Yan F, Wang W, Ying H, Li H, Chen J and Xu C: S149R, a novel mutation in the ABCD1 gene causing X-linked adrenoleukodystrophy. Oncotarget 8: 87529-87538, 2017.

16. Eichler F, Duncan C, Musolino PL, Orchard PJ, De Oliveira S, Thrasher AJ, Armant M, Dansereau C, Lund TC, Miller WP, et al: Hematopoietic stem-cell gene therapy for cerebral adrenoleukodystrophy. N Engl J Med 377: 1630-1638, 2017.

17. Shimozawa N, Honda A, Kajiwara N, Kozawa S, Nagase T, Takemoto Y and Suzuki Y: X-linked adrenoleukodystrophy: Diagnostic and follow-up system in Japan. J Hum Genet 56: 106-109, 2011.

18. Ogaki K, Koga S, Aoki N, Lin W, Suzuki K, Ross OA and Dickson DW: Adult-onset cerebello-brainstem dominant form of X-linked adrenoleukodystrophy presenting as multiple system atrophy: Case report and literature review. Neuropathology 36: 64-76, 2016.

19. Li JY, Hsu CC and Tsai CR: Spinocerebellar variant of adrenoleukodystrophy with a novel ABCD1 gene mutation. J Neurol Sci 290: 163-165, 2010.

20. Jiang MY, Cai YN, Liang CL, Peng MZ, Sheng HY,Fan LP, Lin RZ, Jiang $\mathrm{H}$, Huang $\mathrm{Y}$ and Liu L: Clinical, biochemical, neuroimaging and molecular findings of X-linked Adrenoleukodystrophy patients in South China. Metab Brain Dis 30: 1439-1444, 2015.

21. Dubey P, Raymond GV, Moser AB, Kharkar S, Bezman L and Moser HW: Adrenal insufficiency in asymptomatic adrenoleukodystrophy patients identified by very long-chain fatty acid screening. J Pediat 146: 528-532, 2005.

22. Miyoshi Y, Sakai N, Hamada Y, Tachibana M, Hasegawa Y, Kiyohara $Y$, Yamada $H$, Murakami M, Kondou $H$, Kimura-Ohba S, et al: Clinical aspects and adrenal functions in eleven Japanese children with X-linked adrenoleukodystrophy. Endocr J 57: 965-972, 2010.

23. Polgreen LE, Chahla S, Miller W, Rothman S, Tolar J, Kivisto T, Nascene D, Orchard PJ and Petryk A: Early diagnosis of cerebral $\mathrm{X}$-linked adrenoleukodystrophy in boys with Addison's disease improves survival and neurological outcomes. Eur J Pediatr 170: 1049-1054, 2011.

24. Korenke GC, Roth C, Krasemann E, Hüfner M, Hunneman DH and Hanefeld F: Variability of endocrinological dysfunction in 55 patients with X-linked adrenoleucodystrophy: Clinical, laboratory and genetic findings. Eur J Endocrinol 137: 40-47, 1997.

25. Jorge P, Quelhas D, Oliveira P, Pinto R and Nogueira A: X-linked adrenoleukodystrophy in patients with idiopathic Addison disease. Eur J Pediatr 153: 594-597, 1994.

26. Kemp S, Huffnagel IC, Linthorst GE, Wanders RJ and Engelen M Adrenoleukodystrophy-neuroendocrine pathogenesis and redefinition of natural history. Nat Rev Endocrinol 12: 606-615, 2016.

27. Huffnagel IC, van de Beek MC, Showers AL, Orsini JJ, Klouwer FCC, Dijkstra IME, Schielen PC, van Lenthe H, Wanders RJA, Vaz FM, et al: Comparison of C26:0-carnitine and C26:0-lysophosphatidylcholine as diagnostic markers in dried blood spots from newborns and patients with adrenoleukodystrophy. Mol Genet Metab 122: 209-215, 2017.

28. Aubourg P, Adamsbaum C, Lavallard-Rousseau MC, Rocchiccioli F, Cartier N, Jambaqué I, Jakobezak C, Lemaitre A, Boureau F, Wolf C, et al: A two-year trial of oleic and erucic acids ('Lorenzo's oil') as treatment for adrenomyeloneuropathy. N Engl J Med 329: 745-752, 1993

29. Miller WP, Rothman SM, Nascene D, Kivisto T, DeFor TE, Ziegler RS, Eisengart J, Leiser K, Raymond G, Lund TC, et al: Outcomes after allogeneic hematopoietic cell transplantation for childhood cerebral adrenoleukodystrophy: The largest single-institution cohort report. Blood 118: 1971-1978, 2011.

30. Wiesinger C, Eichler FS and Berger J: The genetic landscape of $\mathrm{X}$-linked adrenoleukodystrophy: Inheritance, mutations, modifier genes, and diagnosis. Appl Clin Genet 8: 109-121, 2015.

This work is licensed under a Creative Commons Attribution-NonCommercial-NoDerivatives 4.0 International (CC BY-NC-ND 4.0) License. 